\title{
Relación entre la petrografía del carbón y el contenido de gases en la cuenca de Amagá (Antioquia, Colombia)
}

\author{
Relation Between Coal Petrography and Gas Content in the \\ Amagá Formation (Antioquia, Colombia)
}

Relação entre a petrografia do carvão e o conteúdo de gases
na bacia de Amagá (Antioquia, Colômbia)

Fecha de Recepción: 14 de Marzo de 2014

Fecha de Aprobación: 23 de Mayo de 2014

Jorge Eliécer Mariño-Martínez*

Ángela Mojica-Amaya**

\section{Resumen}

Se investigó la influencia de los macerales con contenido de gas en muestras de tres pozos. Además de estudiar la composición maceral, se determinó la reflectancia de la vitrinita $\left(\mathrm{R}_{\mathrm{o}}\right)$ y los análisis próximos. Se concluyó que el contenido de vitrinita en los pozos excede a los de la inertinita y a la liptinita, alcanzando un valor del $94 \%$, con el rango de carbón bituminoso de alta volatilidad. Por su parte, el contenido de gas va de unos mínimos valores hasta alcanzar los 100 pies $^{3} /$ ton. En los pozos con presencia de intrusivos se observa una dispersión y una relación no lineal entre las variables. Cerca de los intrusivos aumentan los valores de $\mathrm{R}_{\mathrm{o}}$ hasta cerca de 5 . Donde no hay intrusivos se nota un incremento lineal entre contenidos de gas $\mathrm{R}_{\mathrm{o}}$ con el aumento de la profundidad. Los factores más influyentes en la cueca son $\mathrm{R}_{\mathrm{o}} \%$ de vitrinita e intrusiones.

Palabras clave: Petrografía del Carbón, Macerales, Gas Asociado al Carbón, Reflectancia de la Vitrinita, Formación Amagá, CBM.

\section{Abstract}

The macerals influence with the gas content in three wells is investigated. The proximate analysis, and the vitrinite reflectance $\left(\mathrm{R}_{\mathrm{o}}\right)$ were studied, besides the maceral compositiont analysis. The research concluded that vitrinite content in the wells exceed the Inertinite and the liptinite contents, reaching a $94 \%$ value, with a high volatile bituminous coal. The gas content goes from some minimum values, up to $100 \mathrm{SCF} /$ ton. In the wells with intrusive

* Ph.D. Universidad Pedagógica y Tecnológica de Colombia (Sogamoso-Boyacá, Colombia). jorge.marino@uptc.edu.co

** Universidad Pedagógica y Tecnológica de Colombia (Sogamoso-Boyacá, Colombia). angela.mojica15@hotmail.com 
presence, some data dispersion is noticed and the relationship between the variables is non-linear. Close to the intrusive, the $\mathrm{R}_{\mathrm{o}}$ values increase up to near 5. With a depth increase, where there isn't any intrusive, it is noticed a linear increase between the gas content and $\mathrm{R}_{\mathrm{o}}$. The most influential factors in the basin are $\mathrm{R}_{\mathrm{o}}$, the vitrinite $\%$, and intrusions.

Keywords: Coal Petrography, Macerals, Coal Gas Content, Vitrinite Reflectance, Amagá Formation, CBM.

\section{Resumo}

Pesquisou-se a influência dos macerais com conteúdo de gás em amostras de três poços. Além de estudar a composição maceral, se determinou a refletância da vitrinita $(\mathrm{Ro})$ e as análises próximas. Concluiu-se que o conteúdo de vitrinita nos poços excede aos da inertinita e à liptinita, alcançando um valor de $94 \%$, com o rango de carvão betuminoso de alta volatilidade. Por sua parte, o conteúdo de gás vai de uns mínimos valores até alcançar os 100 pés $3 /$ ton. Nos poços com presença de intrusivos se observa uma dispersão e uma relação não lineal entre as variáveis. Cerca dos intrusivos aumentam os valores de Ro até cerca de 5. Onde não há intrusivos percebe-se um incremento lineal entre conteúdos de gás y Ro com o aumento da profundidade. Os fatores mais influentes na bacia são Ro, \% de vitrinita e intrusões.

Palavras chave: Petrografia do Carvão, Macerais, Gás Associado ao Carvão, Refletância da Vitrinita, Formação Amagá, CBM. 


\section{INTRODUCCIÓN}

En Colombia, el Gas Asociado al Carbón (CBM) se perfila como uno de los hidrocarburos no convencionales con mejores posibilidades para su aprovechamiento e industrialización. La exploración de dicho gas está en sus inicios y se hace necesario un conocimiento previo sobre las características del carbón y los parámetros técnicos que determinen su relación con la posible productividad de gas metano.

La estimación del gas recuperable depende de factores tales como: rango del Carbón, contenido de Humedad, contenido de Materia Mineral, composición maceral, profundidad, fracturamiento, volumen, presión de fluido y grado de saturación de gas, entre otros $[8,10$, 7].

El mecanismo de almacenamiento de gas metano asociado al carbón, a diferencia del gas natural, genera algunos inconvenientes en la estimación de recursos; su almacenamiento se produce principalmente por adsorción en las superficies de los poros, pero también está presente, aunque en pequeñas cantidades, como gas libre en las fracturas. En la generación del gas interviene principalmente el rango del carbón que representa el proceso de madurez termal y el tipo de maceral, entre otros factores [7].

Al aumentar la temperatura y la presión, principalmente por procesos de enterramiento, cambia el rango del carbón, que se ve reflejado en la reflectancia del grupo maceral de la vitrinita $\left(\mathrm{R}_{\mathrm{o}}\right)$. La $\mathrm{R}_{\mathrm{o}}$ mide la intensidad o porcentaje de luz reflejada sobre una superficie pulida del maceral vitrinita y es un indicador de rango o calidad del carbón y, por lo tanto, de la capacidad de éste para generar y almacenar metano. Cada tipo de macerales o componentes orgánicos que hacen parte de la masa del carbón almacenan o adsorben diferentes volúmenes de gas, por lo que el análisis de macerales también es un potencial indicador del contenido de gas metano [7, 2]. En la mayoría de los estudios hasta ahora adelantados se ha encontrado que el maceral vitrinita es el más favorable para la acumulación del metano [10]; sin embargo, en algunas cuencas la liptinita muestra una relación más directa con el contenido de gas [8].

En Colombia se han realizado cálculos del potencial de gas metano basados en análisis del rango del carbón, en mediciones directas, análisis inmediatos y profundidad, entre otros $[3,4,6]$. Con respecto al contenido de gas en la cuenca de Amagá, se ha analizado el efecto de las intrusiones ígneas presentes en la zona, pero no se ha relacionado la influencia de los análisis petrográficos (tipo de maceral y reflectancia de la vitrinita) sobre el contenido de gas $[1,5]$.

Por lo anterior,la Uptc sede Sogamoso, a través del "Laboratorio de Materiales, Gas Asociado al Carbón y Shale Gas", realizó un análisis de la información petrográfica obtenida en tres pozos en la cuenca de Amagá [4,1] (Figura 1). Se analizó la relación de los grupos de macerales: vitrinita, liptinita e inertinita, y el valor de la reflectancia de la vitrinita, con el fin de establecer su correspondencia con la capacidad de almacenamiento y generación de metano. Adicionalmente a la petrografía del carbón, se consideró la influencia de otros factores como: análisis inmediatos, profundidad y presencia de intrusiones ígneas en las propiedades de adsorción de los carbones de la cuenca. Durante el estudio no fue posible correlacionar los mantos, razón por la cual la información se presenta en términos de la profundidad. 


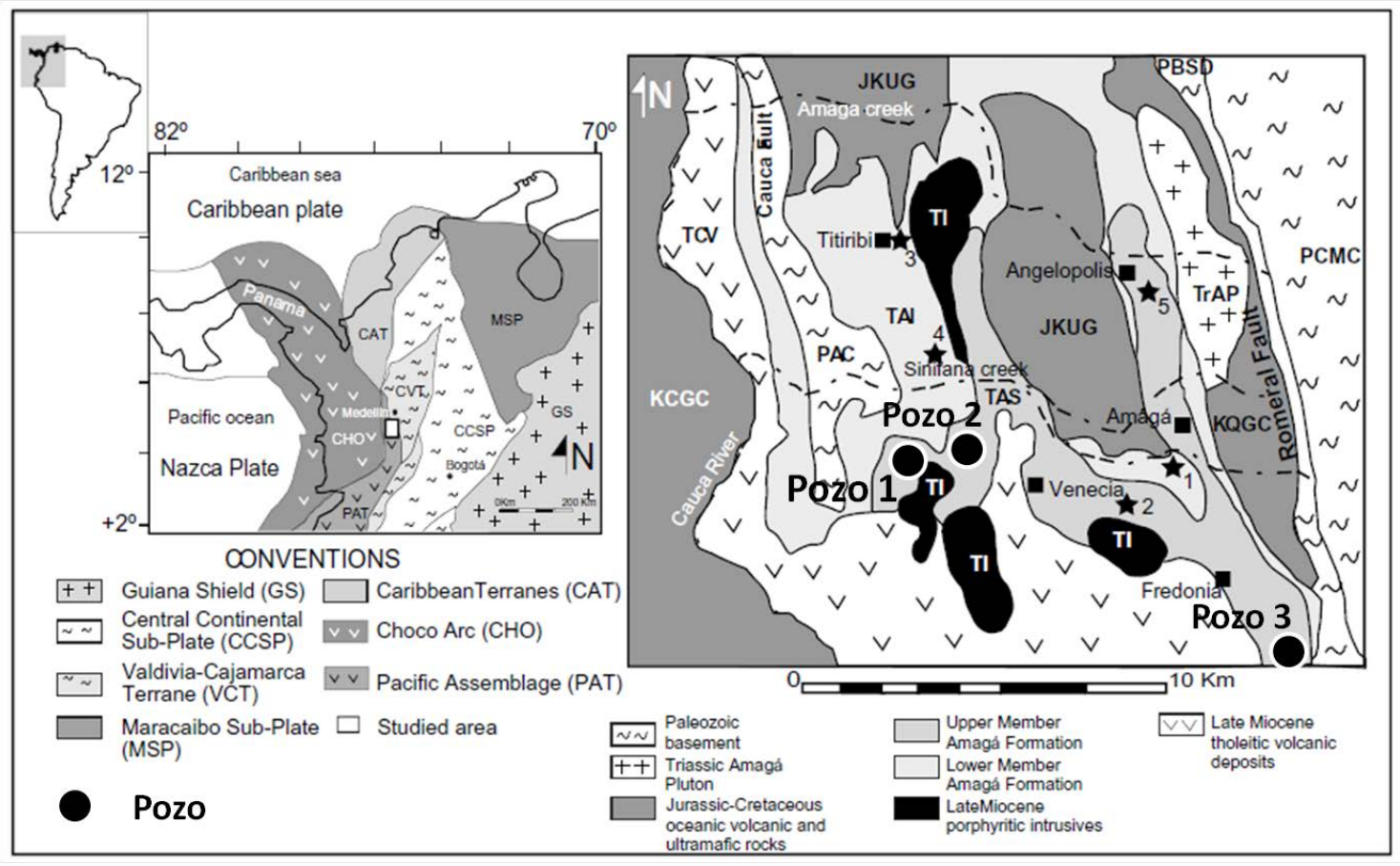

Fig. 1. Geología y ubicación de los pozos de exploración (modificado de [9])

\section{Aspectos geológicos}

La cuenca de Amagá está localizada al oeste de la traza principal del sistema de fallas de Romeral y al este de la falla Cauca; varios factores han hecho de esta una zona muy activa tectónicamente: se encuentra muy próxima al límite entre corteza oceánica y corteza continental, está cerca de la "triple junction" conformada por las placas Caribe, Suramérica y Nazca y está en el límite norte del volcanismo terciario, reciente asociado a la subducción de la placa Nazca [9].

En la cuenca de Amagá afloran un conjunto de rocas sedimentarias con relieve moderado que se encuentran en contacto fallado con rocas volcánicas básicas de la Formación Quebrada Grande, rocas metamórficas (Paleozoico no diferenciado), rocas plutónicas dioríticas y graníticas (Diorita de Pueblito, Plutón de Amagá, respectivamente). El nombre de la Formación Amagá hace referencia a la espesa secuencia siliciclástica y carbonífera de edad Oligoceno Superior a Mioceno Inferior, que reposa discordantemente sobre rocas del Grupo Cajamarca y sobre la Diorita de Pueblito $[5,11]$.

La Formación Amagá está dividida en dos miembros: el miembro inferior, con un espesor aproximado de 294 $\mathrm{m}$, caracterizado por conglomerados mal seleccionados y cuarzosos, sublitoareniscas bien seleccionadas con altos contenidos de cuarzo, limolitas masivas de colores grisáceos y mantos de carbón, y el miembro superior, con un espesor de $228 \mathrm{~m}$, está constituido de wackas líticas, litoareniscas feldespáticas y limolitas de colores verdosos y rojizos (Figura 1) [9]. En el miembro inferior se han identificado entre 2 y 20 mantos de carbón, dependiendo del sector o bloque carbonífero que se estudie.

En la cuenca no se han realizado estudios detallados que permitan valorar el potencial de gas asociado al carbón; solo se cuenta con datos parciales de muestras en frentes de mina y de algunos pozos exploratorios de gas. El contenido de gas para la cuenca de Amagá define unos valores de hasta $100 \mathrm{pies}^{3} /$ ton $[1,5]$.

El rango del carbón de la cuenca es bituminoso alto volátil, y la reflectancia de la vitrinita varía entre 0,4 y 5 [1, 11]. En algunos sectores, como en el Pozo 1, la formación está afectada por fallas de cabalgamiento y por intrusiones tabulares ígneas (silos) de composición andesítica-basáltica paralela a la estratificación, y que a su vez han afectado el rango $\left(\mathrm{R}_{\mathrm{o}}\right)$ y el contenido de gas en los carbones (Figura 2). 


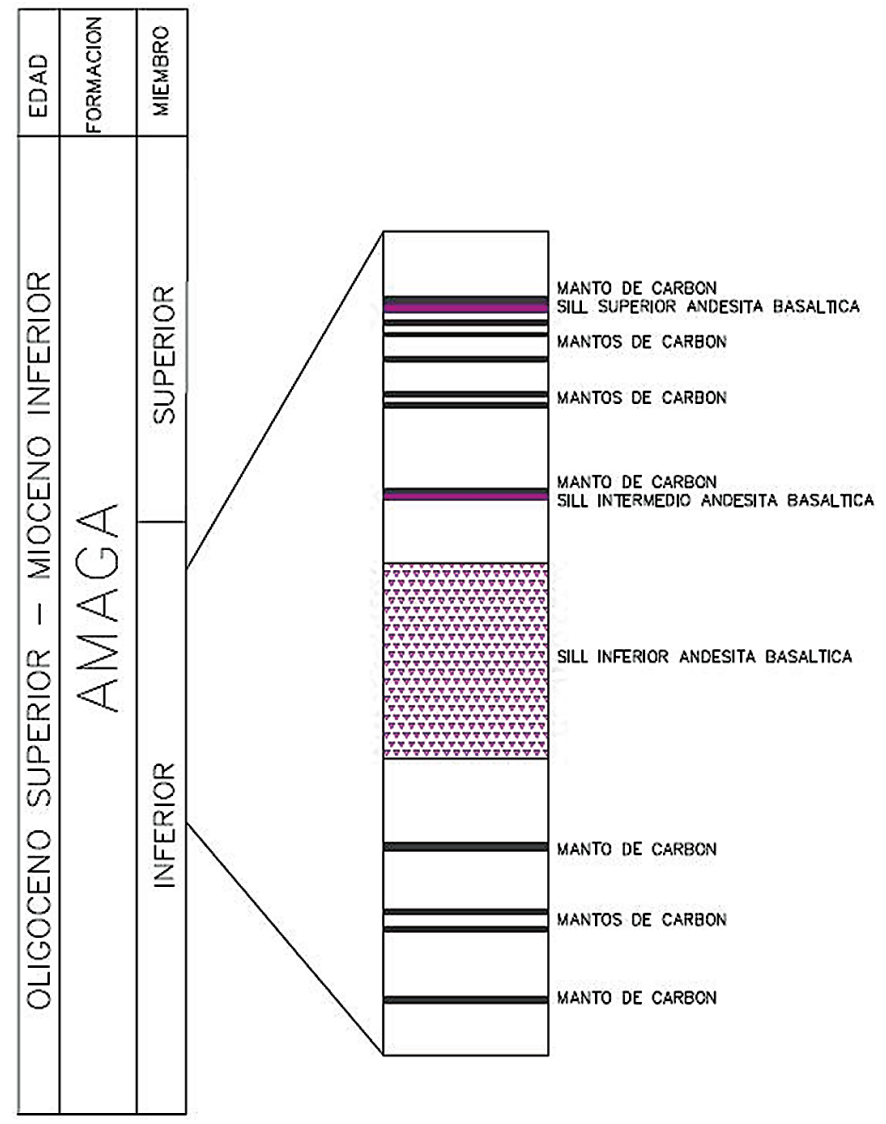

Fig. 2. Descripción estratigráfica de la Formación Amagá en el Pozo 1 (modificado de [1]).Nótese la intercalación de los mantos de carbón con las rocas ígneas (silos).

\section{Métodos y Resultados}

Para el análisis petrográfico de los carbones se utilizó un microscopio Leica DMA4000P, y para medir la reflectancia de la vitrinita $\left(\mathrm{R}_{\mathrm{o}}\right)$ se hizo uso del reflectómetro Tidas MSP. Los análisis inmediatos de los carbones se hicieron en un TGA Leco-14DR del laboratorio de carbones de la Uptc. Para la medición del contenido de gas se utilizaron los equipos de desorción Canisters del "Laboratorio de Materiales Gas Asociado al Carbón (CBM) y Shales Carbonosos (Shale Gas)" de la Uptc-sede Sogamoso.

Para establecer una relación o influencia entre los maceralesy el contenido de gas asociado al carbón presentes en las muestras de tres pozos de exploración de carbón, se graficó la distribución del contenido de los grupos de macerales en porcentaje versus el contenido de gas en pies $^{3} /$ ton por cada muestra (profundidad). Así mismo, se graficó el porcentaje de macerales contra el contenido de materia mineral.
Para relacionar la reflectancia de la vitrinita $\left(\mathrm{R}_{\mathrm{o}}\right)$ con el contenido de gas se graficó el valor de $\mathrm{R}_{0}$ de cada muestra con su contenido de gas. También se evaluaron otros factores que influyen en las propiedades de adsorción de los carbones, analizando los resultados más representativos, tal es el caso del contenido de materia volátil versus contenido de gas, y profundidad versus contenido de gas.

Las Figuras 3, 4 y 5 representan la distribución de los datos al graficar el porcentaje de cada grupo de macerales (vitrinita, liptinita, inertinita) contra el contenido de gas del Pozo1. El análisis de las figuras permitió concluir que, en general, para este pozo no fue posible establecer correlación lineal o directa entre estas variables debido a la alteración que pudo haber tenido el carbón en cuanto a sus características físicas y químicas en su inherente proceso de carbonificación, posiblemente por la presencia de fallas e intrusivos en la cercanía al pozo (Figura 2). Sin embargo, aunque la vitrinita y la liptinita no presentan un patrón de 
relación definido con el contenido de gas, al graficar el contenido de gas versus el porcentaje de inertinita se evidencia que el contenido de gas disminuye con el aumento del porcentaje de inertinita (Figura 5).

En la Figura 6 se grafica el contenido de gas versus la reflectancia de la vitrinita $\left(\mathrm{R}_{\mathrm{o}}\right)$, y se evidencia la tendencia de aumento que tiene este con el aumento en la reflectancia; esto es, a mayor $\mathrm{R}_{\mathrm{o}} \mathrm{o}$ rango, mayor contenido de gas [1]. Los mayores valores

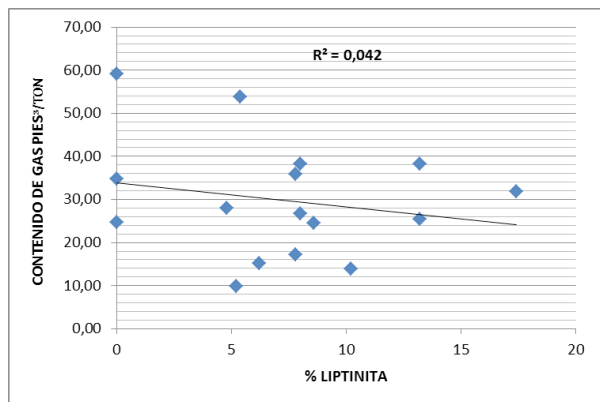

FIG. 3. \% de vitrinita vs. Contenido de gas. Pozo 1.

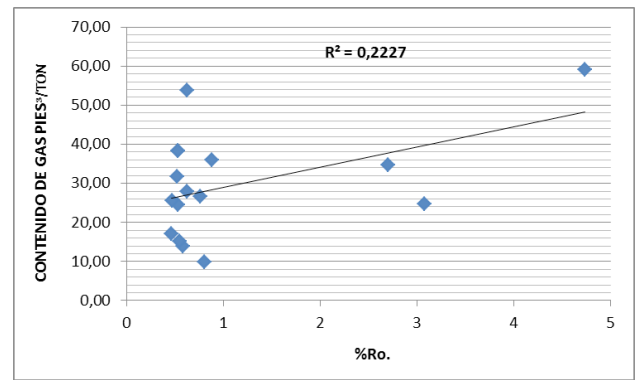

Fig. 5. \% de Inertinita vs. Contenido de gas. Pozo 1

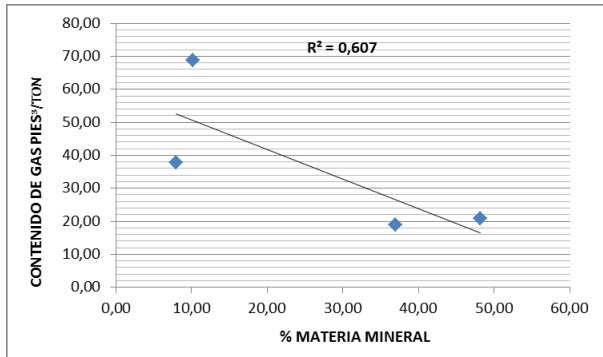

Fig. 7. Materia mineral vs. contenido de gas. Pozo 1

La Figura 7 (Pozo 1) representa un ligero incremento del contenido del gas con el aumento de la materia mineral, cuando se esperaba lo contrario. Lo anterior podría estar relacionado con adición de arcilla de los respaldos durante el muestreo. de reflectancia coinciden con la presencia de tres intrusiones masivas de composición andesíticabasáltica que interestratifican en el pozo 1 con las capas del nivel carbonífero de la Formación Amagá. El efecto de estos cuerpos ígneos incluye aumento en el rango, incremento en la reflectancia de la vitrinita (hasta 4,7\%) en los carbones que están por encima y por abajo (Figura 2), así como también la extensión de aureolas termales.

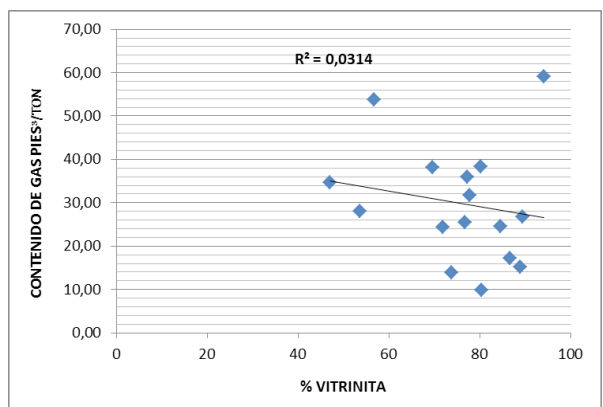

Fig. 4. \% de liptinita vs. Contenido de gas. Pozo 1.

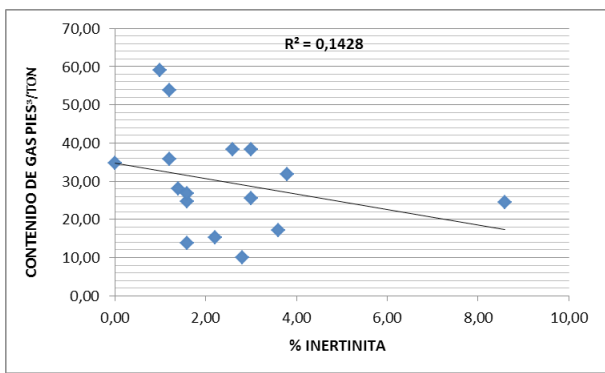

Fig. 6. $\mathrm{R}_{\mathrm{o}}$ vs. Contenido de gas. Pozo 1.

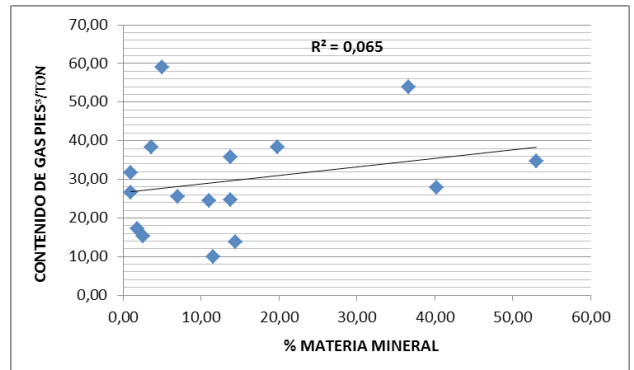

Fig. 8. Materia mineral vs.contenido de gas. Pozo 3

En el Pozo 3 (Figura 8), a pesar de que son solo cuatro muestras, se nota una relación inversa entre la materia mineral y el contenido de gas. El coeficiente de correlación no está tan alto por la posible contaminación del material arcilloso de los respaldos durante la toma de las muestras [10]. 
En las gráficas comparativas en que se representa el contenido de gas, los grupos de macerales y la profundidad para el Pozo 1 se evidencia que la máxima generación de gas se dio en el punto máximo de contenido de vitrinita, con valores de inertinita y liptinita muy bajos, cercanos a cero (Figura 9). Al graficar el contenido de gas ( $\mathrm{pies}^{3} / \mathrm{ton}$ ) versus la materia mineral y la reflectancia de la vitrinita se determina que la materia mineral tiene relación inversa con el contenido de gas y con $\mathrm{R}_{0}$. En el pozo 1 aumenta el contenido de gas con el aumento del $\mathrm{R}_{\mathrm{o}} \mathrm{y}$ disminuye con el aumento de materia mineral (Figura 10).

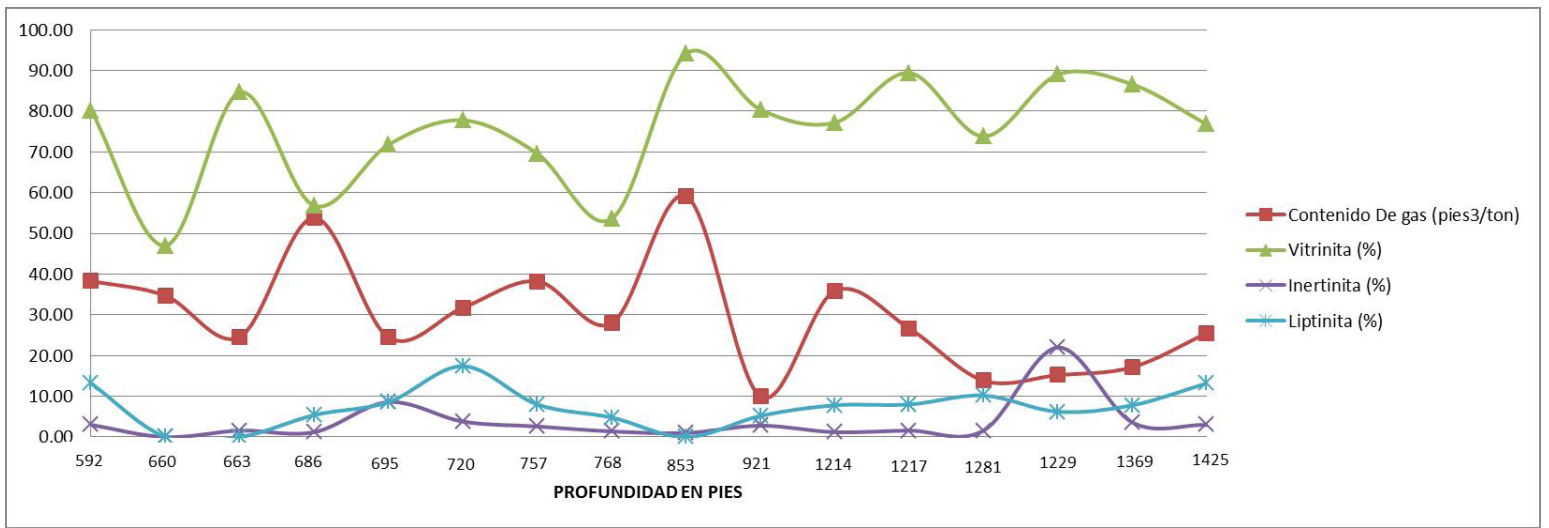

Fig. 9. Pozo 1. Profundidad de la muestra vs. curvas de contenido de gas (pies $3 /$ ton) y $\%$ de grupos de macerales (vitrinita, inertinita y liptinita).

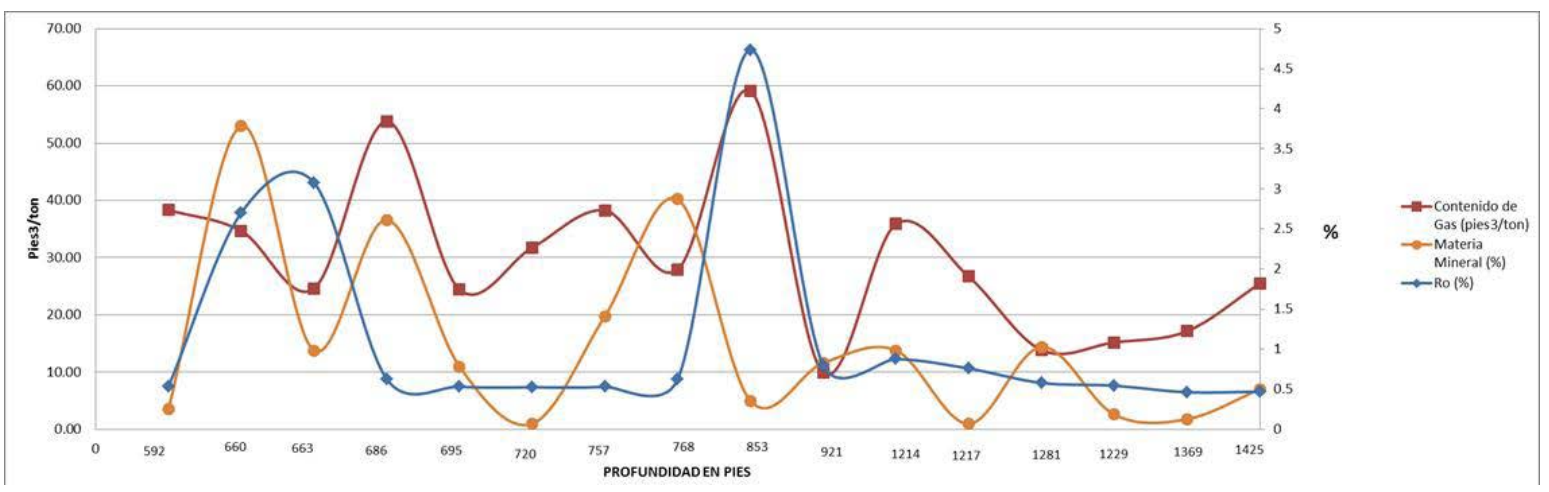

Fig. 10. Pozo 1. Curvas de profundidad vs. contenido de gas, reflectancia de la vitrinita y materia mineral.

La Figura 11 muestra que la materia volátil en el pozo 2 presenta una relación inversa respecto al contenido de gas; lo anterior se debe a que con el aumento del rango del carbón disminuye el contenido de materia volátil, pero aumenta el contenido de gas, como es de esperarse por los mayores rangos o profundidades. La diminución de la materia volátil también se puede explicar por la presencia de intrusivos (Figura 2) [1].
En el Pozo 2, al graficar el contenido de gas versus la profundidad, se define una correlación no lineal con un $\mathrm{R}^{2}=0.227$; esto debido a que el contenido de gas fue afectado por las intrusiones, y los mayores contenidos están cerca al contacto con las andesitas basálticas que actúan como roca sello (Figura 2). Esta anomalía se explica por la presencia de los intrusivos, ya que en los pozos 1 y 3 tampoco fue posible encontrar una relación significativa entre el contenido de gas y la profundidad (Figuras 12 y 13). 


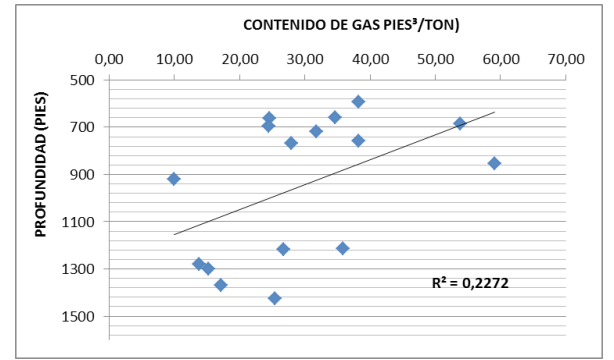

Fig. 11. Materia volátil vs. contenido de gas en el Pozo 2.

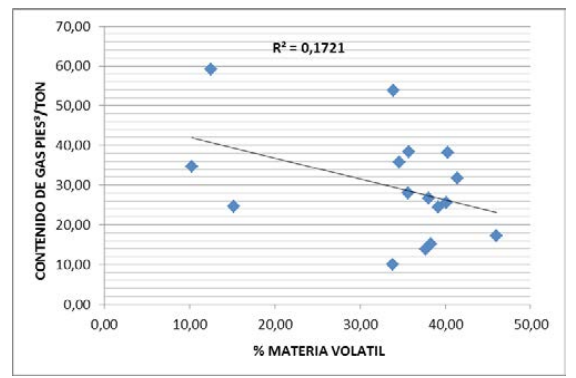

Fig. 12. Profundidad vs. contenido de gas en el Pozo 2.

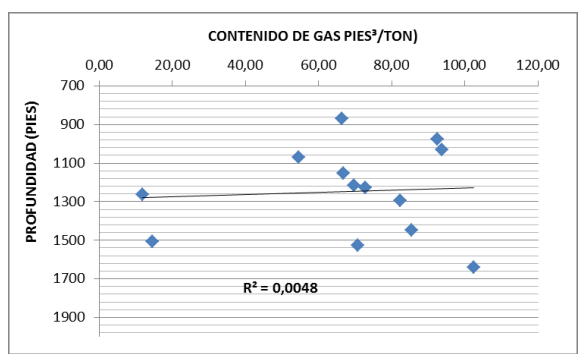

Fig. 13. Profundidad vs.contenido de gas en el Pozo 3.

\section{DisCuSIÓN}

En la cuenca de Amagá hay una buena relación entre el rango del carbón y el contenido de gas; más específicamente, la influencia en el contenido de gas está determinada por unos factores primarios, que incluyen: el grado de carbonificación (rango del carbón), propiedades físicas y químicas del carbón (materia volátil, contenido de cenizas, contenido de humedad), composición maceral y estructura de los poros del carbón, que juegan un papel importante en la formación del gas; sin embargo, los factores secundarios, como concentración del gas por recarga hidrogeológica y formación de gas adicional por factores biogénicos, pueden alterar los contenidos determinados por los factores primarios [7].
Los factores secundarios son: la evolución geológica de la cuenca, la migración de gas, la erosión del paleo-relieve carbonífero, la configuración estructural del carbón y la presencia de intrusivos y fallas que caracterizan la cuenca Amagá. Por tal razón, el rango específico del carbón no siempre se correlaciona con un valor definido en el contenido de gas; esto se debe a que los factores secundarios que rigen la evolución de ciertas partes de la cuenca generan diferencias en el contenido de gas, y, a su vez, permiten evidenciar que la relación petrográfica difiere igualmente en diversos puntos de la cuenca, encontrando en unas muestras de un pozo correlación positiva, y en muestras de otro pozo, una correlación negativa.

En términos generales y de acuerdo con los resultados obtenidos de la petrografía realizada a cada muestra de carbón de los diferentes pozos de la cuenca Amagá, se registra que el contenido de vitrinita excede a la inertinita y a la liptinita, llegando hasta un valor de $94 \%$, con rango de carbón bituminoso alto volátil en su mayoría. El contenido de gas para la cuenca Amagá va de unos mínimos valores hasta los $100 \mathrm{pies}^{3} /$ ton [7]. Se observa dispersión en las gráficas obtenidas de cada porcentaje de macerales (vitrinita, inertinita, liptinita) contra el contenido de gas; no siendo posible establecer una relación lineal entre estas variables, pero sí su caracterización por picos máximos y mínimos, debido a la alteración que pudo haber tenido el carbón por la presencia de fallas e intrusivos en la zona. Los intrusivos alteraron también el valor de reflectancia de la vitrinita $\left(\mathrm{R}_{\mathrm{o}}\right.$ hasta un máximo de $4.7 \%$ (Figuras 6 y 9).

A pesar de la dispersión, en algunas muestras se notó que a mayor porcentaje de vitrinita menor contenido de gas, y a mayor porcentaje de reflectancia de la vitrinita $\left(\mathrm{R}_{\mathrm{o}}\right)$ mayor contenido de gas, ya que en la gelificación bioquímica la compactación toma lugar y la vitrinita es formada disminuyendo los espacios porosos [10]. Esto confirma lo encontrado en cuencas australianas, donde se concluyó que la mayor relación entre la vitrinita y el contenido de gas se debe a que los mantos con más vitrinita tienen un mejor drenaje por el fracturamiento ("cleats") $[8,10]$.

En lo referente a la inertinita y la liptinita, estas debieron expulsar el gas en sus primeras etapas de formación. Ahora bien, cuando el contenido de gas aumenta junto con el contenido de liptinita o inertita, 
se podría estimar que parte del gas escapó de la vitrinita por tener mejor drenaje o fracturamiento $[8,12]$. En cuanto a la materia mineral y la humedad, se encontró que con el aumento de estos parámetros disminuye el contenido de gas. Lo anterior es de esperarse, porque la materia mineral y la humedad ocupan los espacios que le corresponderían a la materia orgánica productora del gas $[7,10]$.

Al comparar los resultados de los análisis inmediatos y la petrografía con los contenidos de gas se muestra dispersión y anomalías, representadas por bajos contenidos de materia volátil y alta reflectancia de la vitrinita $\left(R_{0}>2\right)$ [5]. Para el Pozo 1, dichas anomalías podrían estar relacionadas con las intrusiones presentes en la Formación Amagá y deben analizarse conjuntamente con la geología del área. La relación del mayor contenido de vitrinita y mayor contenido de gas, en algunas muestras, puede indicar que estos carbones tienen un área interna mayor o tienen más microporos, debido a la presencia de gran cantidad de vitrinita.

El resultado inverso del contenido de materia mineral $y$ gas se debe entonces a que la materia mineral tiende a obstruir los poros en la primera etapa de formación del carbón y, así, a retardar la capacidad de retención de gas, agotando el volumen de los poros. En cuanto a la humedad, su tendencia natural es reducir la adsorción de metano y retardar la desorción, por lo que es inversamente proporcional con el aumento de rango, reduciendo la adsorción de metano (Figura 13) [10].

La Formación Amagá es particular, pues presenta influencia volcánica y plutónica, que, a su vez, afecta la deposición y el rango de los carbones. Específicamente en el Pozo 1, la presencia de los intrusivos (silos) aumentó el contenido de gas al aumentar el rango del carbón, y al servir como sello retuvo los gases [5].

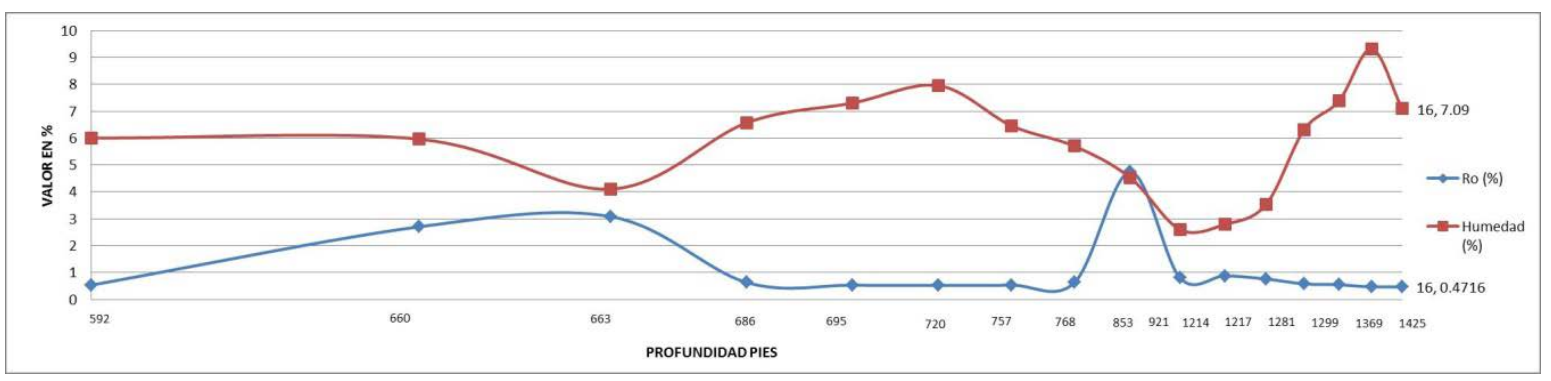

Fig. 13. Relación entre la profundidad, el \% de reflectancia de la vitrinita $\left(R_{\mathrm{o}}\right)$ y el \% de humedad (Pozo 1 )

\section{Conclusiones}

El grupo maceral de la vitrinita es el componente más influyente para la adsorción de gas en la cuenca Amagá y se presenta en mayor porcentaje en todas las muestras analizadas; sin embargo, la influencia de la composición maceral frente al contenido de gas difiere en cada uno de los pozos, debido principalmente a la presencia de factores secundarios como los intrusivos y la migración ocurrida a través de fallas.

El pozo 1 tuvo la mayor influencia de intrusivos y fallas, por lo que no fue posible establecer correlación directa entre variables, dada la alteración que pudo haber tenido el carbón en cuanto a sus características físicas y químicas. Solamente se encontró la relación directa entre el contenido de gas y la reflectancia de la vitrinita.

Los mayores contenidos de gas para la cuenca Amagá están en los carbones Bituminosos Altos Volátiles, con la vitrinita como maceral dominante.

La materia mineral evidencia una relación inversa respecto al contenido de gas con un $\mathrm{R}^{2}=0.6$, mostrando que a medida que ésta aumenta, el contenido de gas disminuye, actuando a su vez como un diluyente.

Las materias volátiles disminuyen a medida que aumenta el grado de carbonificación; esto es más evidente en el Pozo 1 por la presencia de las intrusiones que aumentaron la temperatura y el grado de carbonificación. 
En la cuenca de Amagá se espera que a mayores profundidades aumenten los rangos del carbón y, por lo tanto, los contenidos de gas. Lo anterior va a depender mayoritariamente de la historia de enterramiento y de la evolución tectónica de cada área. En la cuenca las muestras con mayor contenido de gas en el Pozo 1 están influenciadas por el Rango del Carbón; mientras que para el Pozo 3 el contenido de gas depende de la profundidad.

Diferentes influencias pueden ser determinantes en el contenido de gas en variadas áreas e, incluso, dentro de una misma cuenca, dependiendo de factores primarios y secundarios como la composición del carbón, la estructura geológica, la actividad ígnea, los campos de esfuerzos, la hidrodinámica de la cuenca, el enterramiento y la historia térmica. Cada uno de estos factores debe ser analizado por separado para cada cuenca.

\section{Agradecimientos}

Esta investigación fue financiada por Colciencias, la Agencia Nacional de Hidrocarburos y la Universidad Pedagógica y Tecnológica de Colombia, como parte del apoyo al Laboratorio Gases, Carbón, Gas Asociado al Carbón y Shales Carbonosos (Contrato RC N. ${ }^{\circ}$ CT 669/2009). Se agradece, igualmente, el apoyo recibido del señor Carlos Vargas para el análisis de los macerales.

\section{REFERENCIAS}

[1] C. Acuña, J. Mariño, M. Marín, Efectos de los intrusivos ígneos sobre el potencial de gas asociado al carbón (GAC) en el Pozo Venecia 1, cuenca Amagá, Colombia. Resúmenes XII Congreso Colombiano de Geología. Bogotá, Colombia, 2013.

[2] I. Báez, Manual de carbones y coquización. Sogamoso,Universidad Pedagógica y Tecnológica de Colombia. Escuela de Ingeniería de Minas. Facultad Seccional Sogamoso, 2004.
[3] J. E. Mariño, Estudios preliminares del gas asociado al carbón (GAC o CBM) en Boyacá. Trabajo de ascenso en el escalafón. Universidad Pedagógica y Tecnológica de Colombia, Tunja, Colombia, 2010.

[4] L. Mojica, Manual para la medición de gas asociado al carbón (GAC)mediante pruebas de desorción con el equipo cánister. Sogamoso, 2010. Proyecto de grado. Uptc Sogamoso. Escuela de Ingeniería Geológica, 2010.

[5] A. Mojica, Análisis de la información petrográfica de las muestras de carbón en pozos de la cuenca Amagá (Colombia) para determinar su relación con el contenido de gas asociado al carbón. Sogamoso, 2013. Proyecto de grado. Uptc, Sogamoso. Escuela de Ingeniería Geológica, 2013.

[6] L. Mojica, J. Mariño, Estado de la exploración y posibilidades de gas asociado al carbón (GAC) en Boyacá (Colombia). Boletín de Geología UIS, Vol. 35, N. ${ }^{\circ}$ 2, julio-diciembre de 2013.

[7] T. Moore, General Introductionto Coal Bed Methane. In First Annual Illinois Basin Coalbed Symposium. The Midwest PTTC Center. Evansville, Indiana, USA 2004.

[8] B. Scott, P. Crosdale, D. Dingwall, G. Leblang, Coal Petrology and Coal Seam Gas Contents of the Walloon Subgroup-Surat Basin International Journal of Coal Geology 70, 209222, Queensland, Australia, 2007.

[9] J. C. Silva, G. Sierra, L. G. Correa, Tectonic and Climatic Driven Fluctuations in the Stratigraphic Base Level of a Cenozoic Continental Coal Basin, Northwestern Andes. Journal of South American Earth Sciences, N. ${ }^{\circ}$ 26, 369-382, 2008.

[10] R. Walker, M. Glikson, M. Mastalerz, Relations Between Coal Petrology and Gas Content in the 
Upper Newlands Seam, Central Queensland, and Australia. International Journal of Coal Geology 46, 83-92, 2001.

[11] L. Luna, E. Rodríguez, C. Sánchez, G. Renzoni, H. Hernández, J. Maldonado, C. Alba y A. Sanabria, El carbón colombiano-Recursos, Reservas y Calidad, Publicaciones Geológicas Especiales. Ingeominas, Bogotá, 2004.
[12] M. Faiz, A. Saghafia, N. Sherwood, I. Wang, The Influence of Petrological Properties and Burial History on Coal Seam Methane Reservoir Characterization, Sydney Basin, Australia. International Journal of Coal Geology 70, 193208, 2007. 